\title{
Lasers multicolores pour le diagnostic cellulaire
}

\author{
Nelly RONGEAT et Philippe NERIN - Horiba Medical \\ Vincent COUDERC et Philippe LEPROUX - XLIM \\ Guillaume HUSS - Leukos \\ nelly.rongeat@horiba.com
}

Le diagnostic cellulaire est actuellement basé sur les mesures électriques et optiques de multiples paramètres associés aux cellules constituant le sang. L'utilisation de la technique de la cytométrie en flux permet d'obtenir rapidement les caractéristiques de chaque cellule grâce à leur défilement contrôlé devant une ou plusieurs fenêtres $d^{\prime}$ analyse : on mesure ainsi leur taux de fluorescence, leur diffraction ou leur impédance. Pour cela la combinaison de plusieurs sources laser émettant des rayonnements continus à différentes longueurs d'onde est nécessaire mais reste relativement complexe et coûteuse. L'arrivée sur le marché de sources multicolores ultracompactes (de type continuum) permet une amélioration significative du système $d^{\prime}$ excitation optique ce qui va contribuer à obtenir un meilleur dépistage des maladies sanguines. Le choix plus grand des longueurs d'onde et la maîtrise parfaite de leur écartement spectral apportent de nouvelles solutions pour la discrimination cellulaire et cela avec une précision accrue.

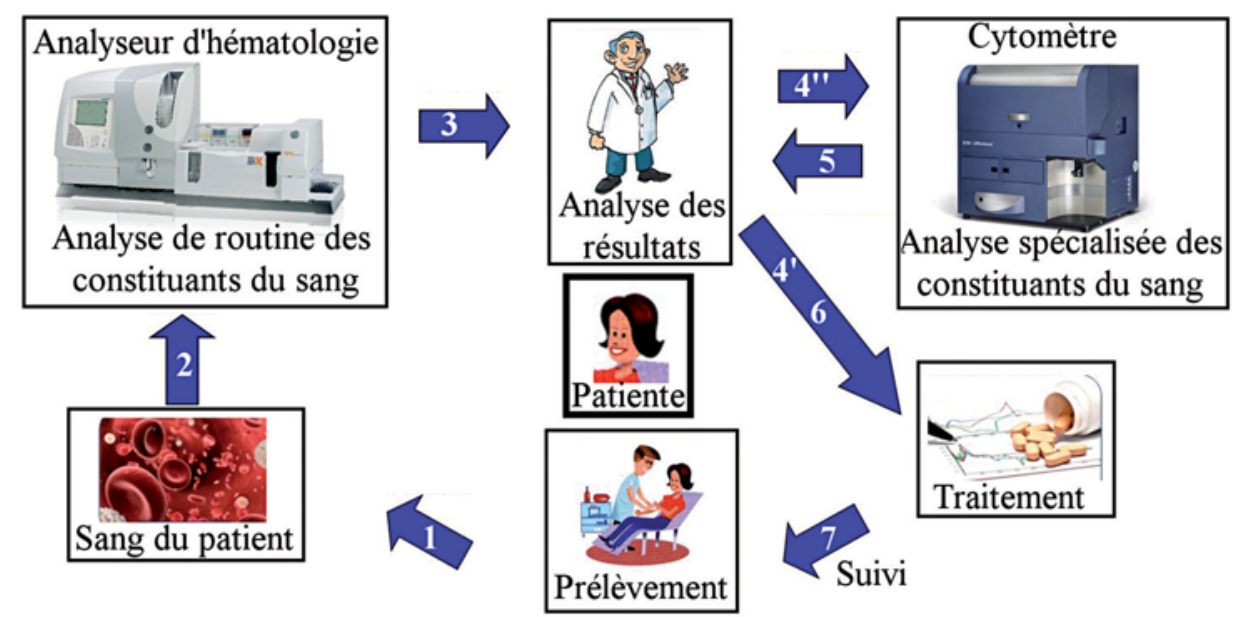

Figure 1. Représentation schématique du parcours de soin.

\section{La situation aujourd'hui}

Un marché très important

En France, les analyses médicales représentent $2,5 \%$ des consommations de soins et de biens médicaux, soit 4,5 Md€ surles 175,7 Md€(2009) que représentent l'ensemble des investissements.

Néanmoins, ces dépenses liées au diagnostic influencent $70 \%$ des décisions médicales constituant ainsi un poste de coût qui pèse fortement sur les collectivi- tés. De plus, ces dépenses ne cessent de croitre dans les pays de l'OCDE avec une progression de près de $10 \%$ entre 2000 et 2003.

Cette tendance globale montre une réelle nécessité d'améliorer le dépistage des maladies afin de pouvoir endiguer, très précocement, toute aggravation et prolifération. Pour cela une amélioration des matériels de diagnostic doit notamment être réalisée. En particulier, des analyseurs d'hématologie, capables en moins de quelques secondes de caractériser plusieurs dizaines de sous-populations cellulaires et d'identifier une cellule anormale parmi plusieurs milliers ou millions, doivent être développés.

\section{Processus de diagnostic et de soin}

L'acte de diagnostic commence le plus souvent par un prélèvement sanguin (figure 1). Celui-ci est analysé par un automate d'hématologie qui fournira dans un premier temps une analyse "de routine» des constituants du sang. Le praticien se livre alors à une première interprétation des résultats:

- si les résultats ne mettent pas en évidence d'anomalie, le patient n'aura pas de traitement,

- si le praticien peut faire un diagnostic à l'aide des résultats, il peut, en général, prescrire un traitement adapté.

Mais dans certains cas, les résultats ne peuvent montrer qu'une anomalie sans pouvoir donner d'information directe sur le diagnostic. Dans ces conditions, le praticien va être obligé de prescrire une nouvelle analyse, cette fois avec un appareil plus complexe qui est appelé un cytomètre. Cetappareil coûteuxn'est 

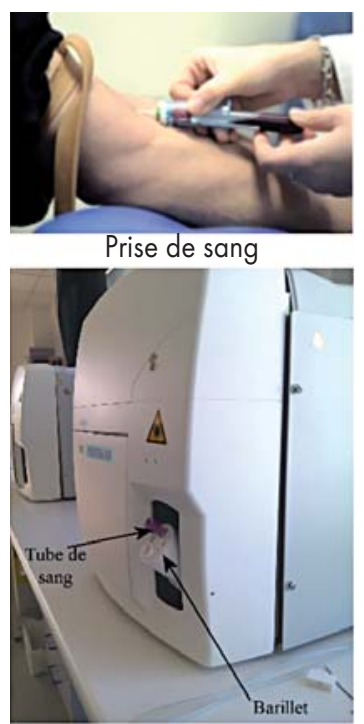

Insertion dans I'analyseur

Figure 2. Photo d'une prise de sang et $d^{\prime}$ un analyseur d'hématologie.

généralement disponible que dans certains laboratoires spécialisés et nécessite l'emploi d'un technicien hautement qualifié. Cela se traduit notamment par le transport des échantillons de sang avec le risque d'une détérioration de leur qualité et un coût d'analyse élevé. Cette seconde étape permet au médecin d'établir un diagnostic définitif et de proposer un traitement adapté. Au cours du traitement, le cycle de prise en charge peut être réitéré, si cela est nécessaire, afin de s'assurer de la bonne efficacité du traitement.

\section{Les appareils de diagnostic}

Les appareils de diagnostic actuels sont particulièrement complexes et permettent de réaliser des tests sanguins ou des analyses pour le suivi de pathologies spécifiques. Ils sont constitués de plusieurs parties relevant toutes d'un domaine technique lla physique, la chimie, la mécanique, la fluidique, l'informatique, l'électronique...).

Concernant les analyseurs d'hématologie, les caractéristiques les plus importantes pouvant actuellement être améliorées sont notamment : la sensibilité afin de dépister très précocement les maladies, la rapidité, la capacité à réaliser un grand nombre de tests simultanément. Le coût de ces appareils doit également être raisonnable afin de permettre à tous d'avoir accès aux soins.

\section{Le principe d'un analyseur d'hématologie}

À la suite d'une prise de sang classique, le tube de sang est introduit dans l'appareil (figure 2). Le sang (composé des cellules et du plasma) est transféré dans le cœur analytique de la machine après cheminement dans différents circuits hydrauliques. Le sang est mélangé avec un réactif puis amené au niveau de la cuve de mesure.

Dans un second temps, les cellules sont focalisées hydrauliquement grâce à des liquides que nous appelons liquides de gainage, et qui permettent de faire défiler les cellules les unes après les autres devant une première fenêtre de mesure électrique puis une seconde fenêtre d'illumination optique (figure 3 ).

Ces mesures donnent des informations sur la taille de la cellule, sur sa granula-

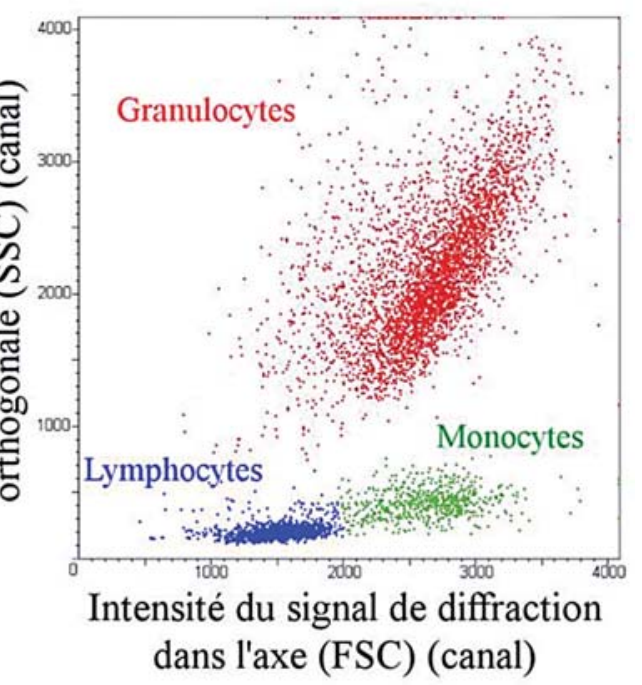

Résultat

Figure 4. Représentation bi-paramétrique ou matrice.

rité, sur son contenu intracellulaire ou sur sa nature. Il est alors possible de les discriminer suivant des catégories et d'afficher ces résultats sur un «simple» hémogramme.

Ces résultats sont ensuite traités par des algorithmes permettant d'obtenir des représentations bi-paramétriques (figure 4), et de classer et comptabiliser les différentes sous-populations cellulaires afin de détecter un éventuel déséquilibre des constituants du sang.

Il est à noter que les analyses optiques dans un analyseur d'hématologie utilisent la plupart du temps une seule source lumineuse (figure 5). Par contre, plusieurs lasers, émettant un rayonnement cohérent à différentes longueurs d'onde, sont généralement intégrés dans les cyłomètres en flux pour obtenir une identification

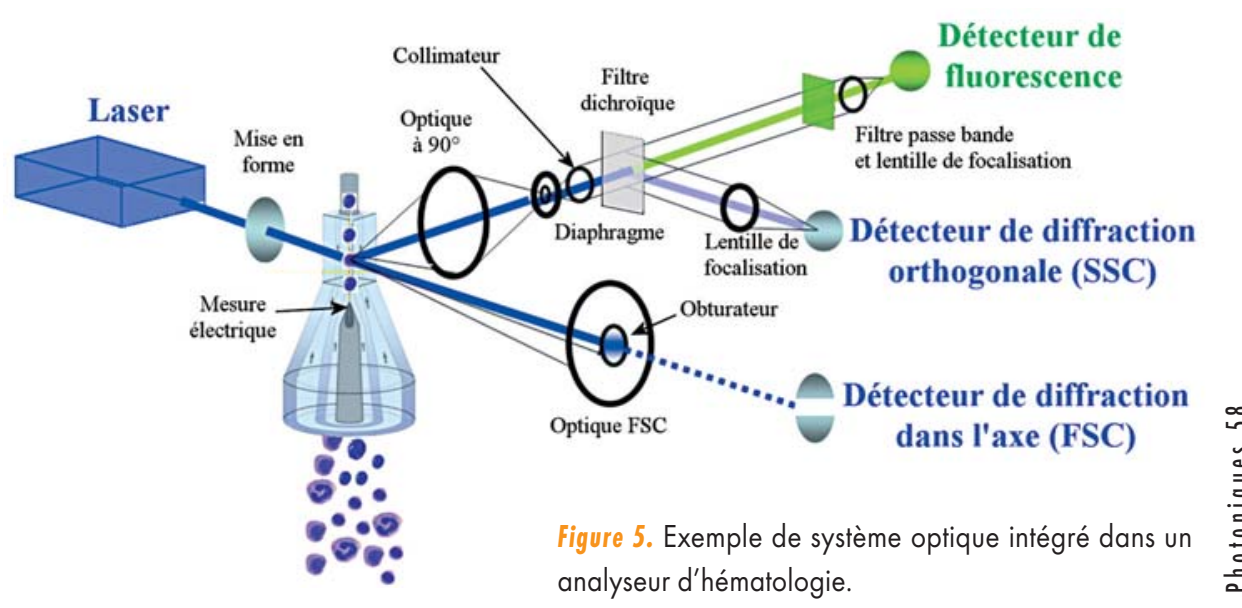




\section{Excitation monolongueur d'onde}

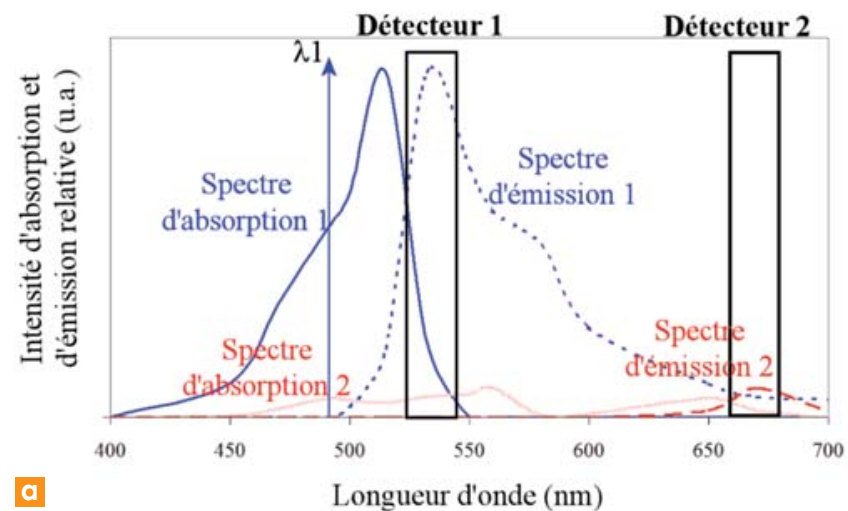

\section{Excitations bilongueurs d'onde contrôlées en intensité}

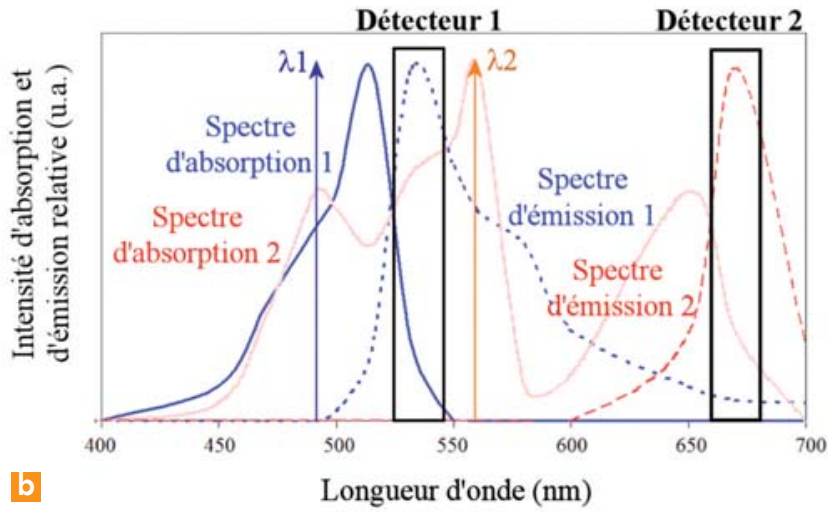

Figure 6. Spectres d'absorption et d'émission de deux fluorochromes. Représentations des longueurs d'onde d'excitation laser, $\lambda_{1}$ et $\lambda_{2}$.

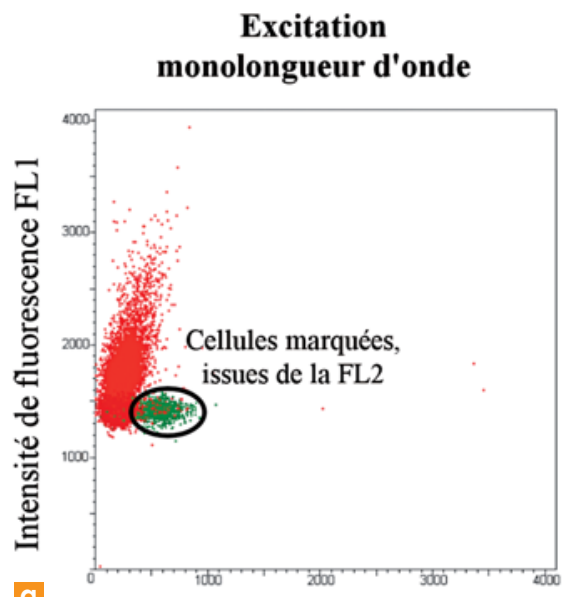

Intensité de fluorescence FL2
Excitations bilongeurs d'onde contrôlées en intensité

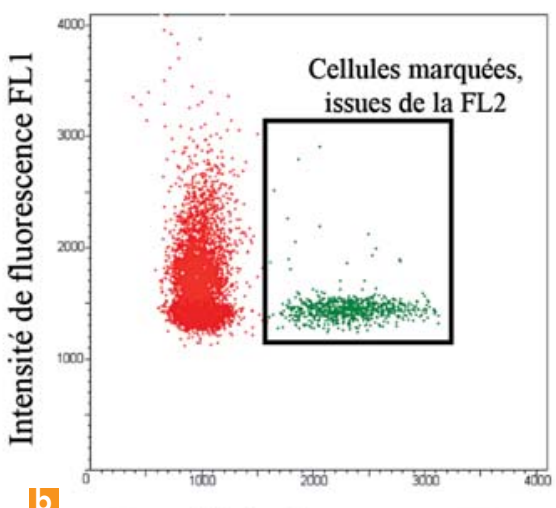

Intensité de fluorescence FL2

Figure 7. Deux excitations combinées permettent de discriminer les éosinophiles et les basophiles parmi les autres leucocytes.

de multiples fluochromes spécifiques de certaines cellules et cela grâce à plusieurs traitements chimiques préliminaires.

\section{Amélioration de la précision du diagnostic cellulaire}

\section{- Grâce à plusieurs sources lumineuses}

L'association de sources lasers émettant des rayonnements lumineux de différentes couleurs et de différentes intensités permet d'améliorer fortement la séparation de populations ou sous-populations cellulaires, surtout quand les fluorescences ont des intensités éloignées. Ceci permet d'ajuster les niveaux de fluorescence des deux fluorochromes utilisés (figure 6).

Ce type de discrimination est basé sur des mesures électriques d'impédance et des mesures optiques de diffraction et de fluorescence. La discrimination des éosinophiles et des basophiles parmi les autres leucocytes /c'est-à-dire les monocytes, neutrophiles, lymphocytes, immatures granuleux, cellules à haute teneur en acides nucléiques) peut être fortement améliorée en utilisant deux excitations combinées grâce à un modulateur acousto-optique (figure 7).

\section{- Grâce à un système d'identification par codage}

La discrimination de deux types de microsphères fluorescentes peut également être obtenue en utilisant une source laser émettant deux radiations synchrones (491 et 532 nm). Le codage est effectué grâce à un modulateur acousto-optique, les modulations de l'intensité sous forme sinusoïdale, à des fréquences $\omega_{1}$ et $\omega_{2}$ sont associées respectivement aux longueurs $d^{\prime}$ onde d'émission laser $\lambda_{1}$ et $\lambda_{2}$. Le codage de chaque signal optique incident permet de discriminer les signaux de fluorescence provenant de différents types de microsphères malgré le recouvrement de leurs spectres de fluorescence (figure 8). Cette nouvelle technique de codage spectral ouvre la voie à de nouveaux axes de recherche et permet d'améliorer la précision du diagnostic dans le domaine de I'hématologie.

\section{Exploitation d'une source laser} multilongueur d'onde pour le diagnostic cellulaire

L'utilisation de plusieurs sources lumineuses au sein d'un système d'analyse engendre de l'instabilité opto-mécanique et les longueurs d'onde fixes des sources lasers ne permettent pas d'exciter les cellules avec la précision spectrale nécessaire. L'utilisation d'un système unique capable d'engendrer un grand nombre de longueurs d'onde (sources blanches cohérentes) permet de contourner efficacement ces problèmes et d'améliorer sensiblement la précision des systèmes d'analyse. Une découpe ultrarapide du spectre d'émission (utilisation d'un modulateur optique) permet d'obtenir un peigne de fréquences ajustable et de gérer, en temps réel, l'amplitude de chaque onde.

Les sources lasers de type super-continuum, basées sur l'utilisation d'un microlaser et d'une simple fibre micro-structurée, 

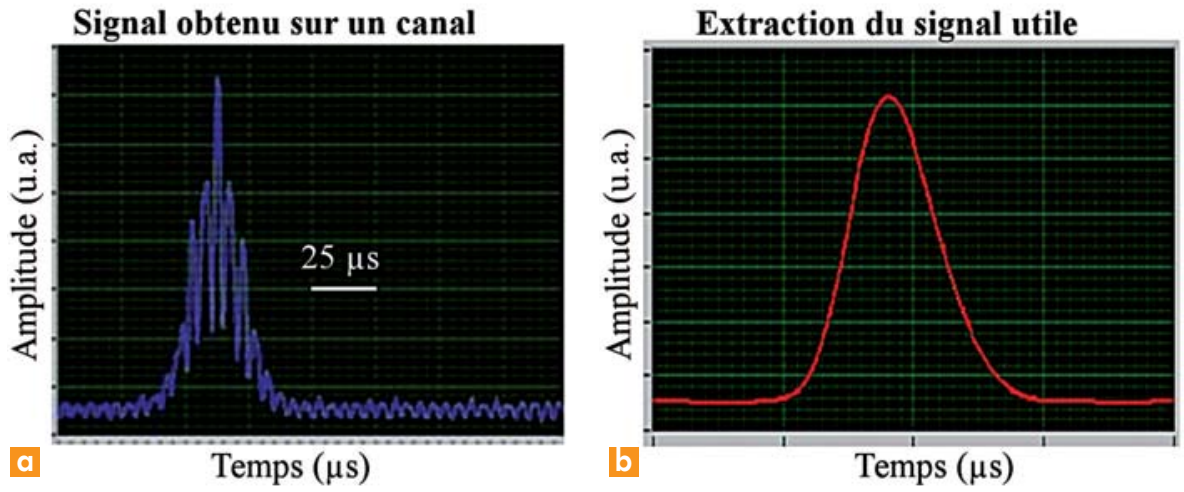

Figure 8. Exemples de (a) signal modulé détecté sur un canal et correspondant à une microsphère, (b) signal utile extrait à partir du signal (a).
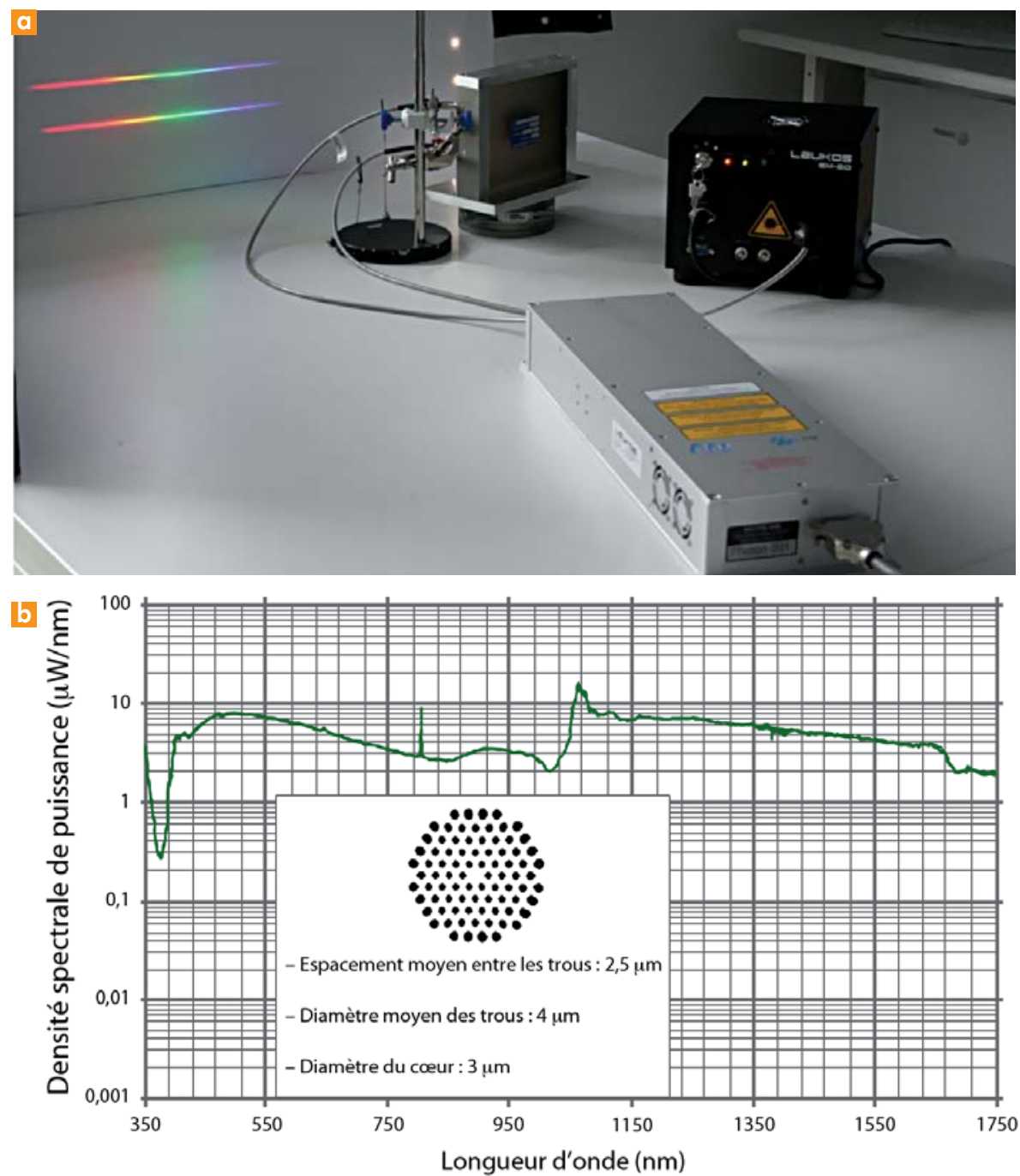

Figure 9. Exemple de supercontinuum (XLIM, Leukos) : (a) le laser utilisé et (b) son spectre d'émission.

constituent donc un outil de choix. Ces émetteurs polychromatiques, développés et commercialisés, dans notre cas, par le laboratoire XLIM et la jeune société Leukos (figure 9), offrent des performan- ces excellentes en termes de stabilité d'amplitude, de largeur spectrale et de densité de puissance. Les systèmes lasers miniatures sont également des systèmes déclenchés. Horiba Medical a donc

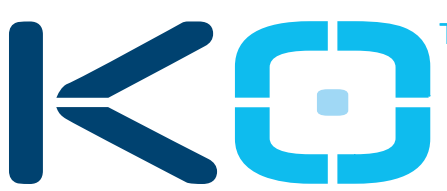

KNIGHT OPTICAL

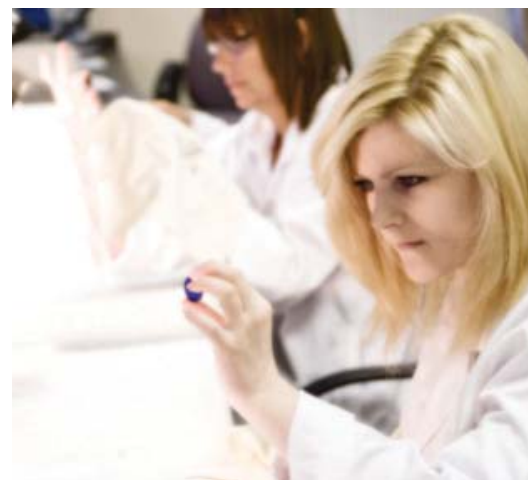

Composants optiques de qualité, opto-mécanique, sous-ensembles
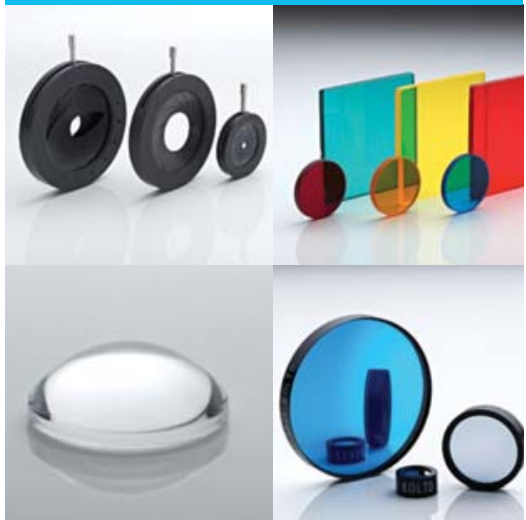

Notre équipe de

conseillers techniques est à votre disposition

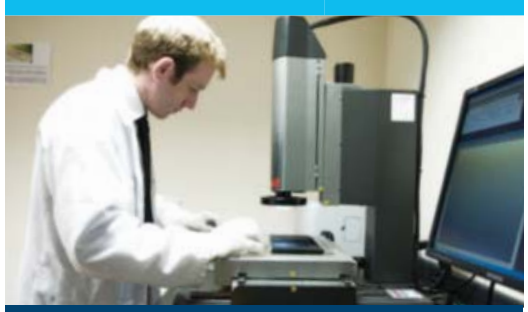

CONTACT

Tél. : +44 1622850614

BertrandMarcillaud@knightoptical.co.uk

knightoptical.com 


\section{Des travaux menés dans le cadre du projet NextGenPCF}

Les résultats présentés dans cet article ont été obtenus lors de la thèse de doctorat de Nelly Rongeat dans le cadre d'une bourse CIFRE et d'un partenariat entre la société Horiba Medical spécialisée dans le diagnostic en hématologie, et l'Université de Limoges (laboratoire XLIM). Ces travaux de recherche se sont déroulés en partie dans le cadre du projet européen NextGenPCF (Next Generation Photonic Cristal Fiber - FP6) qui avait pour but de développer de nouvelles fibres optiques micro-structurées en silice et de démontrer leur utilité dans la réalisation de sources lasers multicolores appliquées au diagnostic cellulaire. Le consortium de NextGenPCF rassemblait dix-huit groupes de recherches industriels et académiques parmi lesquels Horiba Medical (Montpellier), Draka Comteq, Leukos (Limoges), I'Université de Lille (Phlam), I'Université de Limoges (XLIM), I'Université de Jena (Allemagne) ou encore I'Université de Bath (Angleterre).

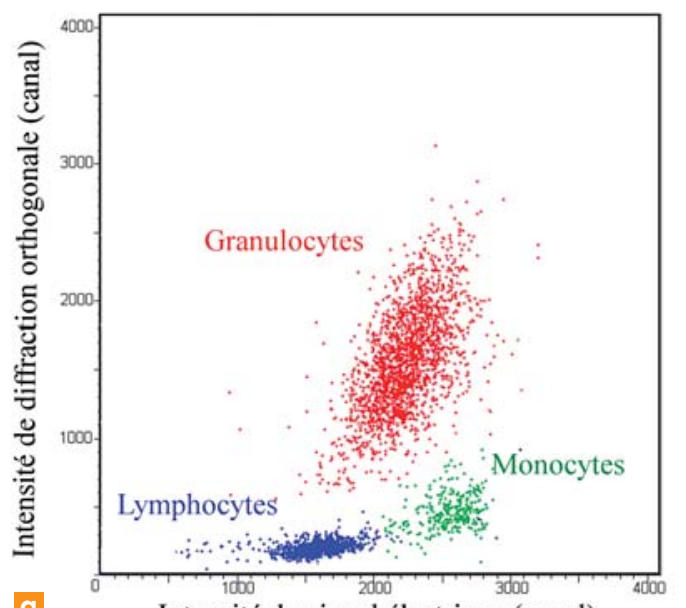

Intensité du signal électrique (canal)

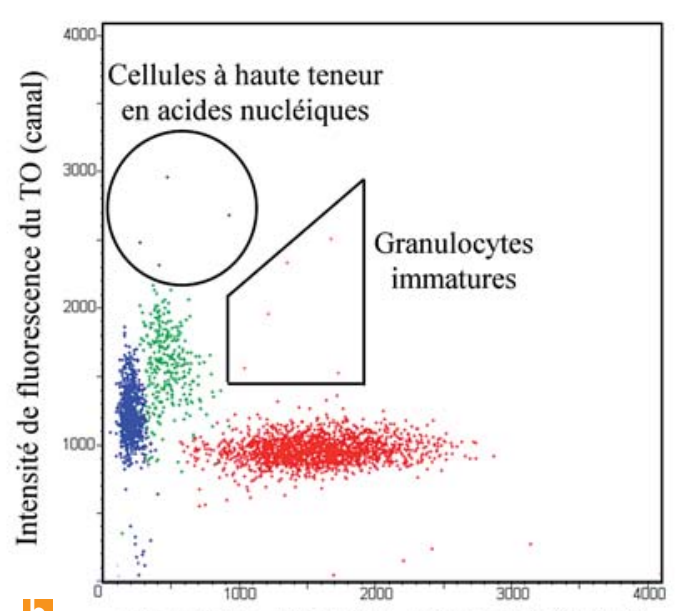

Intensité du diffraction orthogonale (canal) analyses de fluorescence avec un excellent rapport signal sur bruit. La fréquence optimale de ces sources est directement liée à la statistique de passage des cellules dans la fenêtre de mesure et reste dans un ordre de grandeur acceptable pour des sources miniatures déclenchées activement et passivement ( $20 \mathrm{kHz}$ ).

Horiba Medical souhaite poursuivre l'étude de l'implantation des sources polychromatiques dans ses analyseurs. Des tests cliniques systématiques devraient également valider de manière forte ces nouveaux systèmes. Horiba Medical a d'ailleurs effectué une demande de subvention auprès d'OSEO dans le cadre des projets de type ISI (Innovation Stratégie Industrielle) afin de poursuivre ces investigations. Ce projet, dat@diag, a été accepté et a débuté le $1^{\text {er }}$ janvier 2012 et cela en partenariat avec, entre autres, I'Université de Limoges. Il va permettre de poursuivre les efforts de recherche présentés ici pour aboutir à des produits innovants et performants. Ces dispositifs de diagnostic permettront de gagner en précision sur l'aide au diagnostic et le suivi thérapeutique, et donc de détecter plus facilement des maladies spécifiques pour mieux les combattre, afin d'accroître la durée de survie des patients.

vement d'une longueur d'onde dans le bleu (autour de $482 \mathrm{~nm}$ ) a par exemple permis d'obtenir une excellente discrimination des lymphocytes, des monocytes, des granulocytes, des granulocytes immatures et des cellules à haute teneur en acides nucléiques (telles que les lymphoblastes, les monoblastes et les plasmocytes).

\section{Perspectives}

L'utilisation de sources multi-longueurs d'onde dans des analyseurs cellulaires conduit à de réelles avancées en termes de diagnostic de précision. Le coût de ces sources reste tout à fait abordable et permet d'obtenir suffisamment de densité spectrale de puissance pour garantir des

\section{Horiba Medical}

Horiba Medical, dont le siège social est situé à Montpellier, fait partie du groupe japonais Horiba qui compte environ 5000 personnes réparties dans 25 pays dans le monde. Horiba Medical est spécialisée dans la conception et la fabrication de systèmes $d$ 'analyse in vitro appliqués à l'hématologie et la biochimie, et compte 800 personnes dont près de 600 sur le site du siège social. Sur le marché du diagnostic hématologique, et en termes de production d'instruments, Horiba Medical occupe une place prépondérante en Europe et est le $3^{\mathrm{e}}$ acteur mondial. 